\title{
Isolated pulmonary valve infective endocarditis in a middle aged man caused by Candida albicans: a case report
}

\author{
Sudhakar Devathi ${ }^{1}$, Bryan Curry ${ }^{2}$ and Saumil Doshi ${ }^{1 *}$
}

\begin{abstract}
Background: Pulmonary valve endocarditis without the involvement of other valves represents $1.5-2 \%$ of all cases of infective endocarditis. Isolated pulmonary valve endocarditis caused by Candida is extremely rare with only one reported case in the literature and none reported in the United States. Guidelines for management of Candida endocarditis recommend a combination of medical and surgical therapy.

Case presentation: A 61-year-old homeless male presented with fever, cough and shortness of breath. He was urgently intubated for hypoxia. He was initially diagnosed with pneumonia but did not improve with empiric antibacterial therapy. Candida species were isolated from bronchoalveolar lavage fluid and the patient eventually developed persistent C. albicans bloodstream infection. On further workup he was found to have infective endocarditis with a large vegetation across the pulmonary valve. No other valves were involved. He was treated with intravenous antifungal therapy for eight weeks. Valvular surgery was not performed. Follow up echocardiography after completion of therapy did not show any vegetations and the patient clinically improved.

Conclusion: This is the second reported case of isolated pulmonary valve endocarditis caused by Candida and the first to be successfully managed with antifungal therapy alone. Pulmonary valve endocarditis should be considered in cases of pneumonia with Candida and persistent fungemia. While surgery should be considered in all cases of Candida endocarditis, cure may be achieved with antifungal therapy alone.
\end{abstract}

Keywords: Pulmonary valve, Candida albicans, Fungal endocarditis

\section{Background}

Pulmonary valve endocarditis without the involvement of other valves represents $1.5-2 \%$ of all cases of infective endocarditis [1]. Fungal endocarditis is also rare, comprising $<10 \%$ of all endocarditis cases [2]. Despite the development of newer antifungal agents, mortality from Candida endocarditis is estimated to be 30-37\% [3-5]. The Infectious Diseases Society of America 2009 candidiasis guidelines recommend a combined medical and surgical approach for treatment of Candida endocarditis [6]. There has been only one reported case of isolated pulmonary valve endocarditis with Candida which was managed surgically [7]. We report a case of isolated

\footnotetext{
* Correspondence: saumil.doshi@howard.edu

'Division of Infectious Diseases, Howard University College of Medicine, Washington, DC, USA

Full list of author information is available at the end of the article
}

pulmonary valve endocarditis due to Candida managed with medical therapy alone.

\section{Case presentation}

A 61 year-old homeless African-American male was admitted to the intensive care unit with fever, dyspnea, and hemoptysis for four days. He had a history of depression and active intravenous drug use. Physical examination was positive for crackles in both lung fields. He had no track marks on his skin. His white blood cell (WBC) count was $0.3 \times 10^{3} / \mu \mathrm{L}$ with absolute neutrophil count $100 / \mathrm{mm}^{3}$. Urine toxicology screen was positive for opiates and benzodiazepines. His basic metabolic panel was normal but his arterial blood gas (ABG) analysis revealed a $\mathrm{PaO}_{2}$ of $62 \mathrm{~mm}$ $\mathrm{Hg}$ on room air. He was intubated in the emergency room for hypoxia. Computed tomography (CT) scan of the chest revealed large patchy opacities in the right upper and middle lobes consistent with pneumonia and no evidence 
of pulmonary embolism (Figure 1A). Empiric vancomycin and piperacillin-tazobactam were started. His initial blood cultures and serum HIV ELISA antibody were negative. Three tracheal aspirate specimen smears were negative for acid-fast bacilli.

Bronchoscopy performed on hospital day three showed black secretions from the right lower lobe. He was started on Micafungin after bronchoalveolar lavage cultures grew Candida albicans and Candida glabrata. Transbronchial biopsy was not performed due to friable mucosa. Trans-thoracic echocardiogram on hospital day five revealed no valvular abnormalities or vegetations. Because of persistent fever and hypoxia, a second bronchoscopy was performed on hospital day eight, which still showed black secretions; bronchoalveolar lavage cultures grew Candida glabrata. Two sets of blood cultures collected on hospital day twelve grew Candida albicans. A central venous catheter that had been placed on admission was removed; culture of the catheter tip showed no growth. Amphotericin B lipid complex was initially added to Micafungin. After his creatinine rose from 0.9 to 4.5 $\mathrm{mg} / \mathrm{dL}$, this was switched to Liposomal Amphotericin B; his creatinine normalized to $1.1 \mathrm{mg} / \mathrm{dL}$ by hospital day thirty. Additionally, his WBC count rose to $4.9 \times 10^{3} / \mu \mathrm{L}$ with $68 \%$ neutrophils calculated on an automated differential with no interventions other than treating his underlying sepsis. The patient defervesced three days after amphotericin B was added and Micafungin was discontinued. However, repeat blood cultures on hospital day fourteen again grew Candida albicans. A trans-esophageal echocardiogram (TEE) performed on hospital day sixteen showed a $1.5 \mathrm{~cm}$ mobile mass on the pulmonary valve extending from the right ventricular outflow tract across the pulmonary valve into the pulmonary artery (Figure 2A).

A diagnosis of isolated pulmonary valve endocarditis secondary to Candida was made. Cardiothoracic surgery was consulted; they advised medical therapy and later re-evaluation as the patient was a high-risk candidate for surgery. Blood cultures obtained on hospital day 16 had no growth. After a tracheostomy was performed, the patient was transferred to a nursing home to complete liposomal amphotericin B for a total of eight weeks. His repeat $\mathrm{CT}$ chest at the time of discharge showed complete resolution of pulmonary infiltrates (Figure 1B) and he was weaned to tracheal mask. Lifelong suppressive oral fluconazole therapy was recommended. After eight weeks of antifungal therapy, a repeat TEE did not show any pulmonary valve vegetation (Figure $2 \mathrm{~B}$ ). The patient clinically improved, his tracheostomy was closed, and he was discharged back to the shelter. Six months after discharge, the patient was asymptomatic and doing well. He had stopped taking his oral fluconazole despite recommendations for lifelong suppressive therapy.

\section{Discussion}

This report illustrates an unusual case of isolated pulmonary valve endocarditis with Candida in an intravenous drug user that was managed successfully with medical therapy alone. Isolated pulmonary valve endocarditis is rare [8]. Endocarditis in an intravenous drug user usually involves the tricuspid valve, but a structurally normal pulmonic valve is rarely the only one affected [9]. Possible reasons for this are twofold. First, the lower pressure gradient across the pulmonic valve leads to less shear stress compared to the other valves $[10,11]$. This causes less valvular damage, making it less vulnerable for endocarditis. Second, valvular abnormalities (congenital or acquired) are less common in the pulmonary valve.

An autopsy analysis of nine cases of isolated pulmonary valve infective endocarditis revealed seven cases had congenital heart disease and five cases had septic pulmonary emboli [12]. It is unclear if our patient had a primary Candida pneumonia leading to endocarditis or a Candida blood stream infection with endocarditis and septic pulmonary emboli. We do not have a trans-bronchial biopsy to confirm a diagnosis of Candida pneumonia,

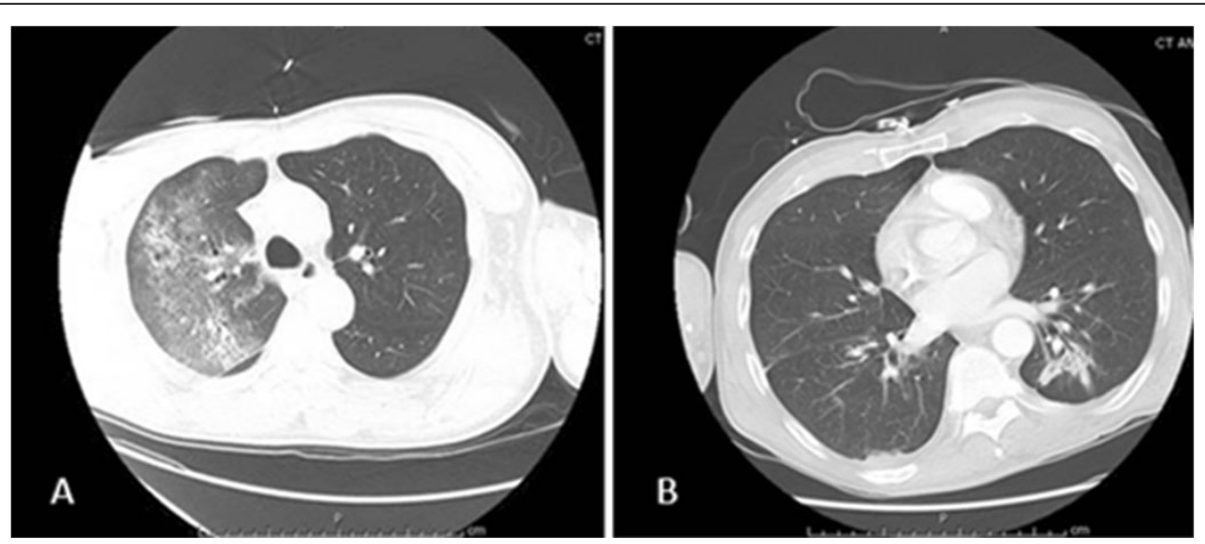

Figure 1 Computed tomography of the chest. A. Patchy opacities inthe right lung. B. Resolution of the opacities after intravenous Amphotericin B. 

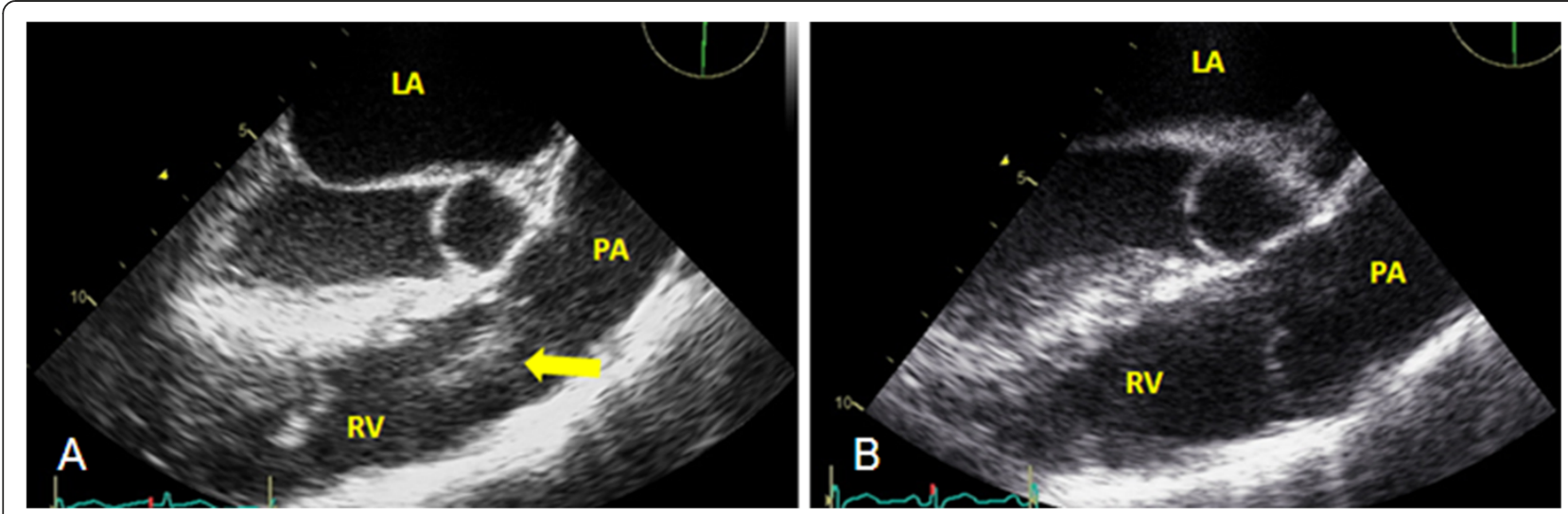

Figure 2 Transesophageal echocardiogram. A. Pulmonary valve vegetation B. No vegetation after four weeks of Amphotericin B.

which is by itself, is rare [13]. A patient with suspected Candida pneumonia should have a work-up for right-sided endocarditis.

The only other reported case of isolated pulmonary valve endocarditis due to Candida described a 66-year-old man with C. parapsilosis fungemia. In that case, a twostage operation was performed: initial resection of the pulmonary valve leaflets with vegetations and subsequent valve replacement two years later [7]. Current endocarditis guidelines recommend initial or induction therapy with amphotericin B with or without flucytosine with surgical removal of vegetation, followed by chronic suppressive therapy with oral fluconazole [14]. Although he had a large fungal vegetation, our patient did not have any other indications for surgery, such as congestive heart failure, valve dehiscence, or peri-valvular abscess [14]. His chest radiography did not reveal evidence of new septic emboli once therapy was started and his blood cultures sterilized soon after the diagnosis of endocarditis was made. There is conflicting data on outcomes associated with the combined medical and surgical approach versus medical therapy alone [15]. Because patients with Candida endocarditis often have other co-morbidities that affect the decision to operate and their outcomes, the optimal management is unclear. However, there have been reports of successful therapy of Candida endocarditis with antifungal medical therapy without surgical intervention [16].

Fluconazole and amphotericin B both target ergosterol, a component of the fungal cell membrane. Treating Candida endocarditis can be difficult because Candida species can form biofilms on native and prosthetic heart valves that can lead to the poor antifungal activity of these agents. The resistance occurs through multiple mechanisms, including decreasing the cell membrane content of ergosterol. The newer antifungal agents, such as amphotericin B lipid formulations and the echinocandins, exhibit novel activity and may be more efficacious against Candida biofilms [17]. Combination therapy with echinocandins and lipid formulations of amphotericin B may be able to treat Candida endocarditis while limiting the emergence of resistance [18]. This may obviate the need for surgical removal of the fungal vegetation.

\section{Conclusion}

Persistent Candida in respiratory cultures without other etiologies for sepsis may be a sign of right-sided Candida endocarditis. Aggressive medical management of this rare condition may be successful with newer antifungal agents that have activity against Candida biofilms. Medical therapy without surgical intervention deserves consideration, especially in cases where surgery may not be an option. However, such patients should be monitored closely for signs of treatment failure on medical therapy alone, such as septic embolization.

\section{Consent}

Written informed consent was obtained from the patient for publication of this case report and accompanying images.

\section{Abbreviations \\ $\mu \mathrm{L}$ : Microliter; $\mathrm{mm}^{3}$ : Cubic millimeter; $\mathrm{PaO}_{2}$ : Partial pressure of oxygen; ABG: Arterial blood gas; CT: Computed tomography; HIV: Human immunodeficiency virus; ELISA: Enzyme-linked immunosorbent assay; Mg/dL: Milligram per deciliter; TEE: Trans-esophageal echocardiogram.}

\section{Competing interests}

The authors declare that they have no competing interests.

\section{Authors' contributions}

Both SD and SD were involved in managing the patient, conducted the literature review, and drafted and edited the manuscript. BC interpreted the echocardiography findings and participated in drafting the manuscript. All authors read and approved the final manuscript.

\section{Author details}

${ }^{1}$ Division of Infectious Diseases, Howard University College of Medicine, Washington, DC, USA. ²Division of Cardiology, Howard University College of Medicine, Washington, DC, USA. 


\section{References}

1. Deng H, Ma Y, Zhai H, Miao Q: Surgical valve repair of isolated pulmonary valve endocarditis. Interact Cardiovasc Thorac Surg 2013, 16:384-386.

2. Ellis ME, Al-Abdely H, Sandridge A, Greer W, Ventura W: Fungal endocarditis: evidence in the world literature, 1965-1995. Clin Infect Dis 2001, 32:50-62.

3. Baddley JW, Pappas PG: Antifungal combination therapy: clinical potential. Drugs 2005, 65:1461-1480.

4. Baddley JW, Benjamin DK Jr, Patel M, Miro J, Athan E, Barsic B: Candida infective endocarditis. Eur J Clin Microbiol Infect Dis 2008, 27:519-529.

5. Lefort A, Chartier L, Sendid B: Diagnosis, management and outcome of Candida endocarditis. Clin Microbiol Infect 2012, 18(4):E99-E109.

6. Pappas PG, Kauffman CA, Andes D, Benjamin DK Jr, Calandra TF, Edwards JE Jr, Filler SG, Fisher JF, Kullberg BJ, Ostrosky-Zeichner L, Reboli AC, Rex JH, Walsh TJ, Sobel JD: Infectious diseases society of America. Clinical practice guidelines for the management of candidiasis: 2009 update by the infectious diseases society of America. Clin Infect Dis 2009, 48(5):503-535.

7. Uchida W, Hirate Y, Ito H, Kawaguchi O: Two-stage operation for isolated pulmonary valve infections endocarditis with Candida parapsilosis. Interact Cardiovasc Thorac Surg 2013, 17(2):426-427.

8. Gonzalez-Juanatey C, Testa-Fernandez A, Lopez-Alvarez M: Isolated pulmonary native valve infectious endocarditis due to Enterococcus faecalis. Int $J$ Cardiol 2006, 113:E19-E20.

9. Frontera JA, Gradon JD: Right-side endocarditis in injection drug users: review of proposed mechanisms of pathogenesis. Clin Infect Dis 2000, 30:374-379.

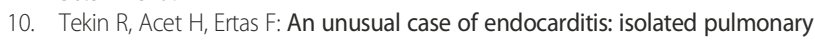
valve endocarditis in patient with patent ductus arteriosus. $J$ Med Cases 2012, 3(6):340-343.

11. William F, Armstrong MD, Thomas Ryan MD: Feigenbaums's Echocardiography. 7th edition. 2009.

12. Vaideeswar P, Jawale RM, Tullu M: Isolated infective endocarditis of the pulmonary valve: an autopsy analysis of nine cases. Cardiovasc Pathol 2009, 18(4):231-235.

13. Haron E, Vartivarian S, Anaissie E, Dekmezian R, Bodey GP: Primary Candida pneumonia.Experience at a large cancer center and review of the literature. Medicine 1993, 72:137-142.

14. Baddour LM, Wilson WR, Bayer AS, Fowler VG Jr, Bolger AF, Levison ME, Ferrieri P, Gerber MA, Tani LY, Gewitz MH, Tong DC, Steckelberg JM, Baltimore RS, Shulman ST, Burns JC, Falace DA, Newburger JW, Pallasch TJ, Takahashi M, Taubert KA: Infective endocarditis: diagnosis, antimicrobial therapy and management of complications: a statement from the committee on rheumatic fever, endocarditis and Kawasaki disease. Circulation 2005, 111:e394-e434.

15. Steinbach, Perfect JR, Cabell CH, Fowler VG, Corey GR, Li JS, Zaas AK, Benjamin DK Jr: A meta-analysis of medical versus surgical therapy for Candida endocarditis. J Infect 2005, 51(3):230-247.

16. Talarmin JP, Boutoille D, Tattevin P, Abgueguen P, Ansart S, Roblot F, Raffi F: Candida endocarditis: role of new antifungal agents. Mycoses 2009, 52(1):60-66.

17. Kuhn DM, George T, Chandra J, Mukherjee PK, Ghannoum MA: Antifungal susceptibility of Candida biofilms: unique efficacy of amphotericin B lipid formulations and echinocandins. Antimicrob Agents Chemotherapy 2002, 46(6):1773-1780.

18. Pai MP: Antifungal combinations against simulated Candida albicans endocardial vegetations. Antimicrob Agents Chemother 2009, 53(6):2629-2631.

doi:10.1186/s12879-014-0557-5

Cite this article as: Devathi et al:: Isolated pulmonary valve infective endocarditis in a middle aged man caused by Candida albicans: a case report. BMC Infectious Diseases 2014 14:557.

\section{Submit your next manuscript to BioMed Central and take full advantage of:}

- Convenient online submission

- Thorough peer review

- No space constraints or color figure charges

- Immediate publication on acceptance

- Inclusion in PubMed, CAS, Scopus and Google Scholar

- Research which is freely available for redistribution

Submit your manuscript at www.biomedcentral.com/submit
C Biomed Central 